LETTER TO JMG

\title{
Identical large scale rearrangement of mitochondrial DNA causes Kearns-Sayre syndrome in a mother and her son
}

\author{
G Puoti, F Carrara, S Sampaolo, M De Caro, C M Vincitorio, F Invernizzi, M Zeviani
}

J Med Genet 2003;40:858-863

M itochondrial disorders are clinical phenotypes associated with abnormalities of the terminal component of mitochondrial energy metabolism - that is, oxidative phosphorylation. Oxidative phosphorylation is carried out in the inner mitochondrial membrane by the four enzyme complexes (I-IV), of the respiratory chain plus the adenosine triphosphate (ATP) synthase complex (complex V). All these complexes, except complex II, which is entirely nucleus encoded, contain both nucleus and mitochondrion encoded subunits, and their biosynthesis also requires co-operation between the nuclear and mitochondrial genomes. Because of this dual genetic control, oxidative phosphorylation diseases can be due to mutations either in mitochondrial DNA (mtDNA) or nuclear DNA genes. Pathogenic mutations of mitochondrial DNA include either large scale rearrangements, which are usually sporadic, or point mutations and micro-rearrangements, which are usually transmitted through the maternal lineage. However, these concepts have recently been challenged by the identification of one family in which a de novo microdeletion in a mtDNA gene was detected in a patient's mitochondrial genome, which had clearly been inherited from the proband's father ${ }^{1}$ and of a family with a mother to offspring transmission of a single mtDNA deletion. ${ }^{2}$

We present here a case of maternal transmission of a single heteroplasmic deleted mtDNA species in two subjects, both affected by Kearns-Sayre syndrome (KSS).

\section{CASE REPORTS}

\section{Patient 1}

A woman with KSS (subject I-1 in fig 1A) died at 48 years of age of respiratory insufficiency. She was the second of four uneventful pregnancies from healthy non-consanguineous parents. At the age of 14 years, she noted the presence of bilateral eyelid ptosis and ophthalmoparesis. Over the following 4 years, both symptoms steadily progressed and she developed bilateral hearing loss, ataxia and proximal limb muscle weakness. At the age of 27 years, she received a heart pacemaker because of several brief episodes of unconsciousness, associated with atrioventricular block of variable severity, documented by repeated electrocardiograms. Bilateral photophobia ensued 1 year later. She developed overt diabetes mellitus (basal glycemia $232 \mathrm{mg} \%$ ), which required insulin treatment from 41 years of age. Clinical examination at 34 years of age disclosed mild cognitive deterioration, cerebellar ataxia, bilateral ptosis, ophthalmoplegia, bilateral hearing loss, dysarthria, dysphagia, and reduced tendon reflexes. Proximal and distal limb muscles were weak against moderate resistance. Touch sensation was normal. Fundus oculi examination revealed a pigmentary retinopathy. Audiometry confirmed severe bilateral sensorineural hearing loss. Cerebrospinal fluid analysis demonstrated increased protein concentration $(105 \mathrm{mg} / \mathrm{dl}$, normal range 15-45). Muscle specific creatine kinase (CK) was moderately elevated in blood (671 IU/l, normal range

\section{Key points}

- A mother with Kearns-Sayre syndrome and her son with a similar, although milder, clinical presentation, carried an identical, large scale heteroplasmic rearrangement of mitochondrial (mt) DNA in muscle and blood lymphocytes.

- The rearrangement was present in two forms: a $m+D N A$ deletion in skeletal muscle, and a combination of partially deleted and partially duplicated m+DNA molecules in blood. Accumulation of partially deleted mtDNAs in muscle can explain the progressive myopathy found in both probands.

- Our work provides additional evidence that mother to offspring transmission of mtDNA deletion/duplication is indeed possible, although we do not know which of the two molecular forms, or both of them, is the transmissible genetic element. The concept that, however low, a recurrence risk is associated with single mtDNA rearrangements should be included in the clinical management and genetic counselling of patients and families.

38-174), indicating sarcolemmal damage. A computed tomography scan of the brain displayed cerebellar atrophy and signs of cerebral white matter involvement. Electromyography showed myogenic abnormalities and a muscle biopsy showed several ragged red, cytochrome c oxidase (COX) negative fibres (fig 1B). The patient married a healthy man and gave birth to three children: two clinically normal girls and one affected boy. The pedigree is shown in fig $1 \mathrm{~A}$.

\section{Patient 2}

A 19 year old male (subject II-3 in fig 1A), the affected son of individual I-1 (patient 1), developed bilateral eyelid ptosis during infancy, followed by progressive bilateral ophthalmoplegia. No other neurological symptom was reported during childhood and adolescence. Clinical examination showed the presence of bilateral ptosis, complete external ophthalmoplegia, moderate, generalised hypotonia, and reduced tendon reflexes. Cerebellar ataxia and muscle weakness were both absent. Fundus oculi examination and electroretinogram revealed an initial pigmentary retinopathy. Audiometry demonstrated mild bilateral sensorineural hearing loss.

Abbreviations: ATP, adenosine triphosphate; $\mathrm{CK}$, creatine kinase; COX, cytochrome c oxidase; KSS, Kearns-Sayre syndrome; mtDNA, mitochondrial DNA; PEO, progressive external ophthalmoplegia; RCS, revised Cambridge sequence 
Laboratory analyses showed moderately high CK levels (551 IU/l) and basal hyperglycaemia (132 mg\%, normal range 60-120). Brain magnetic resonance imaging was normal. The electrocardiogram revealed an incomplete right bundle branch block. The electromyogram was consistent with a primary myopathy. A few ragged red, COX negative fibres were present in the muscle biopsy (fig 1B).

The two elder sisters of individual II-3 (subjects II- 1 and II-2) are two clinically normal women, 28 and 26 years old, respectively. Neurological and general physical examinations were normal in both.

\section{MATERIALS AND METHODS}

\section{Morphological and biochemical analyses}

Morphological analysis of skeletal muscle, and biochemical assays of the individual respiratory complexes and of citrate synthase on muscle homogenate were carried out as described previously. ${ }^{3}$

\section{Molecular genetic analysis}

Approximately $10 \mu \mathrm{g}$ of total genomic DNA, extracted from muscle tissue or blood lymphocyte buffy coats of the patients and two healthy daughters of patient 1, was digested with the restriction endonucleases $P v u I I$ and SnaBI, which cleave human mtDNA at unique sites: nucleotide (nt) 2652 for $P v u I I$ and nt 10736 for SnaBI. Southern blot analysis was performed using the ECL chemiluminescence based kit from Amersham Pharmacia Biotech Italia (Cologno Monzese, Italy), following the manufacturer's instructions. Two PCR fragments, corresponding to different regions of the human mtDNA revised ${ }^{5}$ sequence, ${ }^{6}$ were amplified and used for the analysis. Probe $\mathrm{IA}, \mathrm{B}$ is composed of two fragments, one (1A) corresponding to $n t 16130-500$ in the D-loop region, the other (1B) to nt4120-5050, encompassing the genes encoding NDl, the tRNAs for Ile, Gln, and Met, and ND2. Probe 2 corresponds to nt 10300-11000m encompassing the ND3, tRNAArg, ND4L and ND4 genes (see fig 2).

The relative amount of rearranged $v$ total mtDNA genomes were calculated by densitometry.

To identify the rearrangement breakpoint region, a PCR fragment was amplified from total genomic DNA samples of the probands, using a pair of oligonucleotide primers corresponding to nt5445-5470 (forward primer) and nt14679-14703 (reverse primer) of the revised Cambridge sequence (RCS) (see also http://www.mitomap.org). The positions corresponding to these two primers are separated by more than $8.0 \mathrm{~kb}$ in the wild type mtDNA, while in the partially deleted mtDNA species they are separated by only $776 \mathrm{bp}$. This difference in size determines a preferential amplification of the fragment corresponding to the deleted mtDNA species, which was in fact the only detectable DNA product of the PCR. To show that mtDNA was present in all samples, we also amplified,using suitable primers in some experiments, such as the one shown in fig 1A, a 475 bp band that corresponds to an mtDNA region outside the rearrangement (nt3125-3600). Automated sequence analysis of this fragment was performed on an ABI 3100 Analyzer, using the ABI Prism ${ }^{\circledR}$ BigDye $^{\mathrm{TM}}$ Terminator kit (both Applera Italia, Monza, Italy).

\section{RESULTS}

No defect of respiratory chain enzymes was detected in the muscle homogenates of the two probands. This result is not uncommon in subjects with KSS or progressive external ophthalmoplegia (PEO) and is supported by the limited number of ragged red, COX negative fibres detected in the muscle biopsy of both individuals.

A first Southern blot analysis was performed on $P v u I I$ digested DNA samples, using probe $1 \mathrm{~A}, \mathrm{~B}$, which is composed of equimolar amounts of two PCR fragments, IA and 1B, corresponding to mtDNA regions that are usually not included in large scale rearrangements of mtDNA in humans $^{78}$ (see fig 2A). The unique PvuII restriction site is located outside the arc between the D-loop and the alleged origin of replication of the light strand $\left(\mathrm{O}_{\mathrm{L}}\right)$, where the vast majority of the large scale rearrangements of mtDNA has been reported to occur $^{7}$ (fig 2A). Digestion with PvuII linearises the wild type mtDNA molecule to a single $16.5 \mathrm{~kb}$ DNA fragment. As a result, a single hybridisation band is obtained by Southern blot analysis, as shown in blood DNA samples from the two healthy daughters of patient 1 (elder sisters of patient 2) (fig 2A). However, the same analysis revealed an identical, abnormal pattern in the samples from the two patients. As shown in fig 2B (panel a) two hybridisation bands were visualised in samples extracted from either muscle or blood. The slow migrating band corresponds to the wild type mtDNA species, which is approximately $16.5 \mathrm{~kb}$ in size, while the fast migrating band correspond to a smaller mtDNA species of approximately $8.0 \mathrm{~kb}$.

The percentage of heteroplasmy of the aberrant mtDNA molecules was $46 \%$ and $34 \%$ in the muscle samples of patients 1 and 2, respectively. The percentage in blood was $51 \%$ in patient 1 , and $22 \%$ in patient 2 .

When the same blot was hybridised with a probe corresponding to a region presumably contained within the rearrangement (probe 2 , see fig $2 \mathrm{~A}$ ), the analysis revealed the presence of a single $16.5 \mathrm{~kb}$ hybridisation band in all samples, corresponding to linearised wild type mtDNA (fig. 2B, panel b). This experiment confirmed that the rearrangement included the region of mtDNA corresponding to probe 2 .

PCR based amplification of the mtDNA region flanking the breakpoint gave a DNA fragment of 776 bp in blood (fig 1A) and muscle samples (not shown) from the probands. No fragment was obtained from DNA samples extracted from blood (fig 1A), epithelial cells of the urinary tract, epithelial cells from oral mucosa, and hair follicles of the two healthy siblings, suggesting that no transmission of the rearranged mtDNA species occurred in these individuals. In contrast, a 475 bp fragment corresponding to a mtDNA region outside the rearrangement could be amplified to a comparable level in all samples (fig 1A). Sequence analysis of the breakpoint region revealed that the rearranged mtDNA species were identical in both muscle and blood DNA from the affected mother and son. The mtDNA rearrangement comprised the loss of a 8522 bp region, (nt5782-14304) of RCS, encompassing 10 tRNA genes and the following structural genes: COI, COII, COIII, ATP6, ATP8, ND3, ND4L, ND4, ND5, and part of ND6. The rearrangement occurred across two direct repeats of five nucleotides: GCTTC. These repeats are present in the wild type mtDNA at the boundaries of the deleted region. Only one repeat was maintained in the rearranged molecule, while the other was part of the deleted sequence. As the 3' distal repeat was located three nucleotides away from the endpoint of the deletion (fig. 2C), these results provide evidence for retention of the 3' distal repeat in the rearranged molecule.

The pattern obtained from PvuII digested samples does not discriminate between a mixture of wild type mtDNA and partially deleted mtDNA species present as single molecules or as deletion dimers from partial duplications, in which a wild type mtDNA molecule is interrupted by the insertion of a partially deleted mtDNA molecule (see fig. 2A). To distinguish between these two different kinds of rearrangement, a second Southern blot analysis was carried out on the same samples, using the restriction endonuclease SnaBI. Like PvuII, SnaBI has a single restriction site in wild type human mtDNA 
A
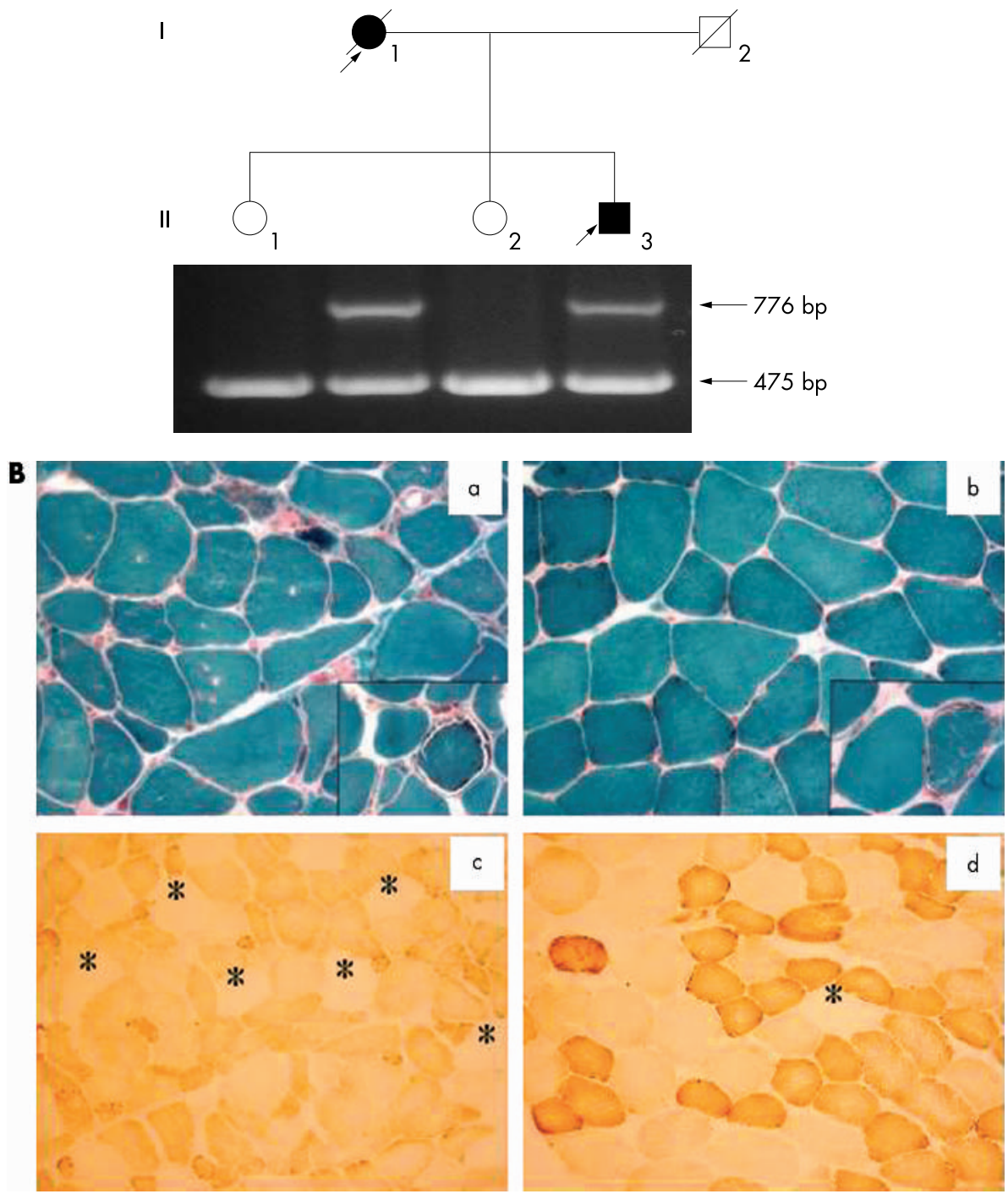

Figure 1 Family structure, PCR based experiments and morphological findings. (A) Pedigree and agarose gel electrophoresis of mtDNA PCR fragments. Black symbols and arrows indicate probands. Each lane of the ethidium bromide stained gel is aligned to the corresponding individuals of the pedigree. The $776 \mathrm{bp}$ band represents a PCR fragment corresponding to a mtDNA region across the breakpoint of the rearrangement. The $475 \mathrm{bp}$ band corresponds to a mtDNA band outside the rearrangement (see Materials and methods for details). (B) Morphological findings in muscle. (a, b) Modified Gomori trichrome. Original magnification $\times 10$; insert $\times 40$. Bar $=40 \mu \mathrm{m}$. (a) Patient 1: severe myopathy with several atrophic and some necrotic fibres. Numerous, isolated ragged red fibres were also seen (see insert). (b) Patient 2: moderate increase in fibre size variability due to the presence of some hypertrophic fibres (mean diameter $80 \mu \mathrm{m}$ ). Sparse ragged red fibres were also found (insert). (c, d) Histochemical reaction to COX. (c) Patient 1: numerous fibres (>15\%) stain poorly or negative to COX $(*)$, and there are numerous atrophic fibres. (d) Patient 2: a few fibers are COX negative (*). A dark staining fibre shows degenerative changes.

(at nt10736). However, the SnaBI restriction site is usually contained, as in these cases, within the rearrangement (fig 2A). As a result, the SnaBI site is absent in partially deleted molecules, while it is present in both wild type and partially duplicated mtDNA species. Digestion with SnaBI linearises both wild type and partially duplicated molecules, but does not linearise partially deleted molecules or deleted dimers. As shown in fig $2 \mathrm{~B}$ (panel $\mathrm{c}$ ), probe $1 \mathrm{~A}, \mathrm{~B}$ revealed an aberrant hybridisation pattern in the SnaBI digested samples from both probands. In muscle samples, a fuzzy, slowly migrating band (band a, arrowed, in fig $2 \mathrm{~B}$, panel c) was present in addition to the $16.5 \mathrm{~kb}$ band, corresponding to wild type mtDNA. Two additional aberrant bands, one migrating faster (band $\mathrm{C}$, arrowed, in fig. $2 \mathrm{~B}$, panel $\mathrm{C}$ ), the other migrating slower (band $b$, arrowed, in fig $2 \mathrm{~B}$, panels $\mathrm{c}$ and d) than the wild type band, were present in blood samples from both probands. Again, the blood DNA samples of the two healthy daughters of proband 1 showed only the wild type band. Based on the above explanation, we interpreted this pattern as follows: the fuzzy hybridisation band " $a$ ", visualised in muscle DNA and also to a lesser extent in blood DNA of patient 1, is composed of nicked, undigested, circular DNA molecules corresponding to deleted mtDNA species, which typically lose their coiled, compact configuration and run as slowly migrating species in the gel. Likewise, the lower band " $\mathrm{C}$ " visualised in blood DNA is also due to the presence of undigested deleted mtDNA species, organised as supercoiled, fast migrating molecules. However, the sharp, slowly migrating band " $\mathrm{b}$ ", which was detected in blood samples, could represent either a partially duplicated species, 
A
wt-m+DNA
$\varnothing-m+D N A$
$8.0 \mathrm{~kb}$
Dup-mtDNA
$24.5 \mathrm{~kb}$
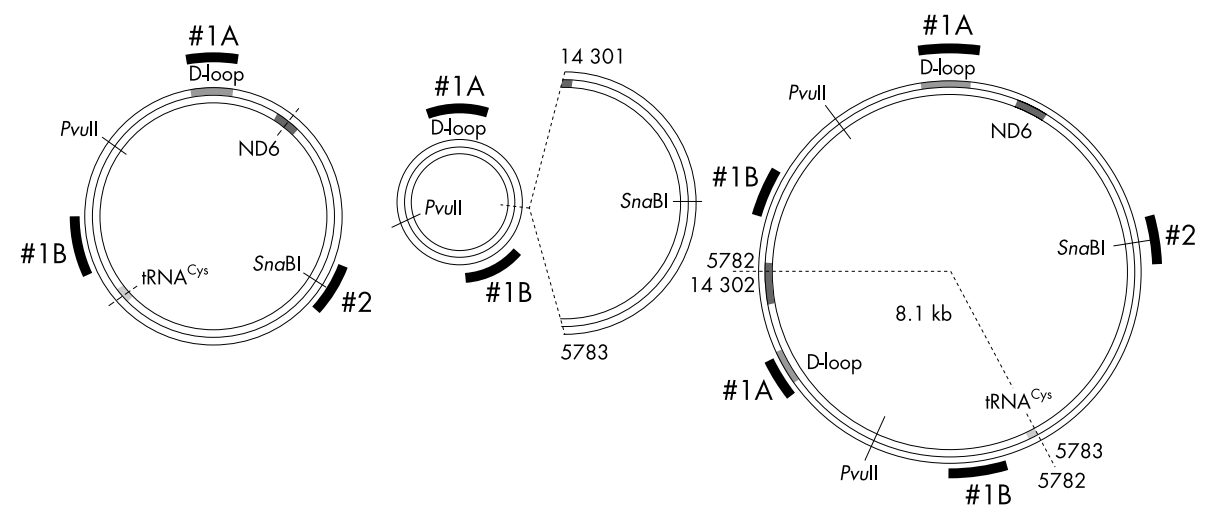

B

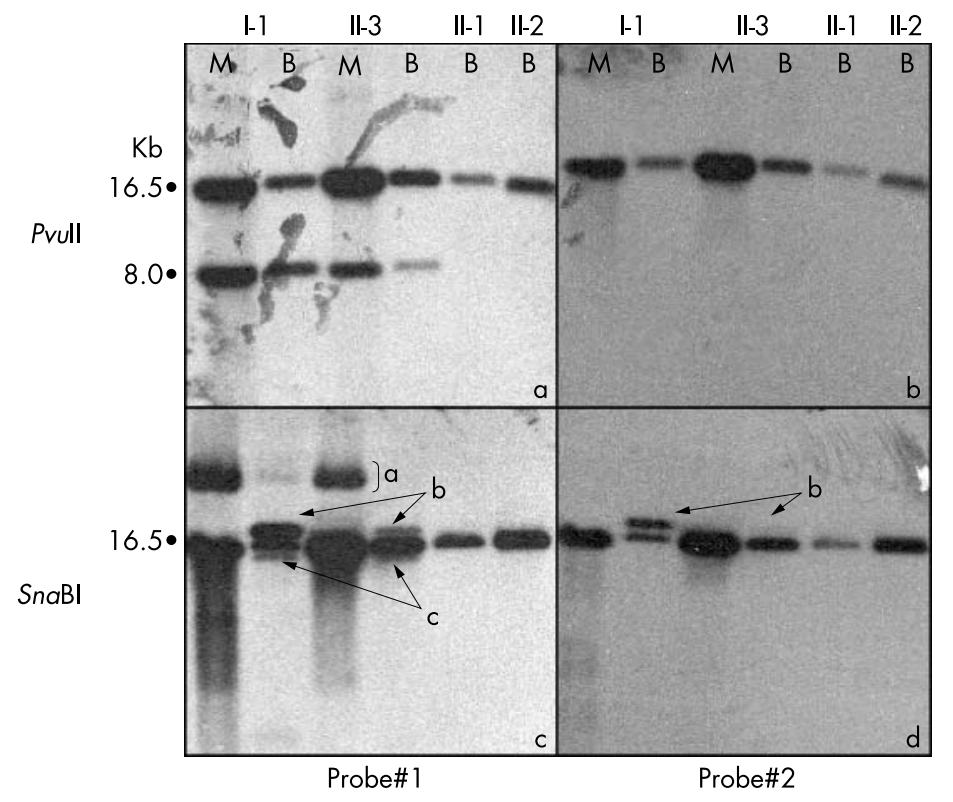

C

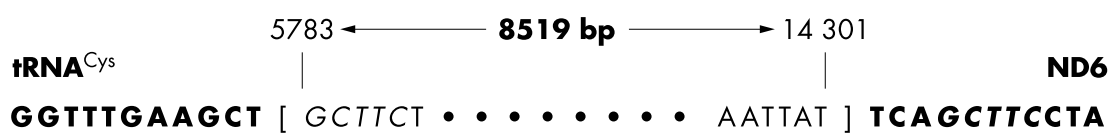

Figure 2 MtDNA analysis. (A) Schematic representation of wild type (wt), partially deleted ( $\Delta$ ), and partially duplicated (Dup) mitochondrial genomes. Solid thick bars indicate the mtDNA regions encompassed by probes 1A, 1B and 2. Dotted lines indicate the boundaries of the rearrangement. Thin bars indicate the position in the mtDNA maps of restriction sites specific to Pvull and SnaBl. Numbers on the maps indicate the nucleotide positions of the rearrangement breakpoints. The D-loop region is indicated by a striped bar. The genes encoding ND6 and tRNA ${ }^{C y s}$, which contain the boundaries of the rearrangement, are also indicated. (B) Southern blot analysis of DNA samples from muscle (M) and blood (B). Patients and maternal relatives are indicated according to the nomenclature in the pedigree tree shown in fig 1A. (a, b) rThe same blot containing Pvull-digested DNA hybridised first with probe IA,B (a) and, after stripping, with probe 2 (b). (c, d) The same blot containing SnaBI-digested DNA hybridised first with probe 1 (c) and, after stripping, with probe \#2 (d). In (c) and (d), the letters $a, b$, c with arrows indicate aberrant bands corresponding to rearranged molecules (see Results). (C) Schematic representation of the breakpoint region. The boundaries of the rearrangement are indicated by brackets. The nucleotide positions corresponding to the breakpoint are shown above the sequence of capital letters representing the mtDNA nucleotide sequence across the breakpoint. The size of the deleted region is indicated between arrows. The tandem direct repeats across the breakpoint are in italic. The genes encoding ND6 and tRNA ${ }^{\text {Cys }}$, which contain the boundaries of the rearrangement, are also indicated.

or a "relaxed" deleted species. To clarify this question, the blot was hybridised with probe 2 (fig. 2B, panel d). As expected, the slowly migrating fuzzy band "a", and the faster migrating band " $\mathrm{C}$ ", were not visualised by probe \#2, indicating that they corresponded to deleted species. By contrast, the slowly migrating, sharp band " $\mathrm{b}$ ", visualised by probe $1 \mathrm{~A}, \mathrm{~B}$ in blood samples, was also detected by probe 2 , indicating the presence of a partially duplicated species. Duplicated molecules were more abundant in the affected mother, but were also detected, although at much lower levels, in her affected son.
Based on these results, we conclude that a single, partially deleted mtDNA was the only aberrant species in muscle, while a mixture of both partially deleted and duplicated mtDNA species was present in blood.

\section{DISCUSSION}

This family was characterised by a mother to son transmission of the same large scale rearrangement of mtDNA. In blood, abnormal mtDNA was present as partially deleted and partially duplicated molecules, but skeletal muscle contained 
only partially deleted mtDNA species. Partially duplicated mtDNAs accounted for approximately half the aberrant species found in blood from both probands. We do not have an explanation for the tissue specific distribution of the different rearrangements in our patients. Partial duplications usually have milder effects on energy metabolism compared with single partial deletions or deletion dimers. ${ }^{9}$ This different pathogenicity has been observed in several cases and it could explain the weak selection against the persistence of these rearrangements in the blood samples of our probands. In contrast, and for yet unknown reasons, the results obtained in skeletal muscle indicate a clonal expansion of a population of partially deleted molecules in this, and possibly in other, post-mitotic tissues. Partial deletions are considered much more deleterious than partial duplications. ${ }^{9}$ The presence of deletions as the only aberrant species in skeletal muscle can account for the typical features and the severity of the clinical phenotype.

KSS is a progressive, multisystem disorder predominantly involving post-mitotic tissues such as the nervous system, the heart and the skeletal muscles. Direct mother to offspring transmission of a partially deleted mtDNA species has recently been described in a single family. ${ }^{2}$ The deletion was found in the muscle of the mother and in the blood of the son. However, as no other tissue was investigated, it cannot be excluded that partially duplicated mtDNA species were present in extra-muscle organs, and notably in the oocytes of the affected mother. In our family, we do not know whether, as previously suggested, partially deleted species were generated from partially duplicated species ${ }^{10-13}$ or, vice versa, the duplicated species were produced by recombination events between a partially deleted and a wild type mtDNA. Both mechanisms could indeed occur in the same cell population, and at the same time or at different times during the lifespan of a particular cell, tissue, or individual.

In spite of the common opinion that mtDNA duplications can be transmitted ${ }^{2} 8$ while deletions cannot, convincing evidence for maternal inheritance of partially duplicated mtDNAs has been reported in only a few maternal lineages. Affected individuals from three families presented with a mild phenotype characterised by diabetes mellitus and deafness. ${ }^{14-16}$ In the first family, what was initially believed to be a huge, $10.4 \mathrm{~kb}$ deletion was later shown to be in fact a partial duplication, ${ }^{16}$ while in the second family a partial duplication was demonstrated to co-exist with a partial triplication of the same mtDNA region. None of the affected individuals in these families had severe neurological symptoms, suggesting that partial duplications are by themselves less pathogenic than partial deletions. ${ }^{9}$ This difference is likely to be due to the fact that the missing genes in the duplicated region are replaced by a complete mtDNA gene repertoire provided by the wild type molecule in cis with the partial duplication (see fig. 2A), resulting in functional complementation. In an additional family, partial duplication was detected in a child with a syndrome resembling severe KSS, whose mother was affected by PEO, but conclusive evidence for vertical transmission of the rearrangement was hampered by limited availability of maternal tissues. ${ }^{17}$ Another report described transmission of a rearrangement from a mother affected by PEO to a child affected by Pearson's syndrome, ${ }^{18}$ but, as in the family initially reported by Ballinger et al, ${ }^{14}$ no further investigation was performed to verify whether the rearrangement was a partial deletion, a partial duplication, or a mixture of the two. This latter work and that by Ballinger et al, illustrate an important, but often overlooked, point, namely the fact that deletions and duplication cannot be distinguished in routine diagnostic procedures. The standard protocols that are used to screen large scale rearrangements of mtDNA are based on Southern blot analysis of PvuII digested DNA samples or, more recently, real time PCR. ${ }^{19}$ Because both methods fail to discriminate between single deletions and duplications, all mtDNA rearrangements are usually considered tout court as "deletions". However, this attribution is completely arbitrary, because of the technical limitations of both methods. Therefore, in spite of the enormous number of observations and studies on mtDNA deletions that have been carried out by many research teams in the last 15 years, the frequency of partial deletions, partial duplications (or triplications ${ }^{15}$ ), or of a combination of the two is virtually unknown in patients affected by mitochondrial disease. Based on the few reports that have specifically addressed this issue, partial duplications, or, more likely, a combination of partially duplicated and partially deleted molecules, ${ }^{10-13}$ could well account for a substantial fraction of the large scale mtDNA rearrangements detected in patients. If this is true, then transmission of partial deletions, partial duplications, or both, is an exceptionally rare event. Nevertheless, our work provides further convincing evidence that, however rare, mother to offspring transmission of rearranged mtDNA species is indeed possible, and that this may also occur between severely affected individuals. As a consequence, the recurrence risk for a mother to offspring transmission of mtDNA deletion/duplication should no longer be considered zero, and must be taken into account in the clinical management and genetic counselling of patients and families. ${ }^{20}$

\section{ACKNOWLEDGEMENTS}

We are indebted to Ms B Geehan for revising the manuscript and Dr E Lamantea for artwork in the preparation of iconography. We thank Professor R Cotrufo for his helpful comments and support to this study.

This study was supported by Fondazione Telethon-Italy (grant no. 1180), Fondazione Pierfranco e Luisa Mariani (Ricerca 2000), Ricerca Finalizzata Ministero della Salute RF-2002/158, and MitEuro network grant from the European Union Framework Program 5.

\section{Authors' affiliations \\ G Puoti, S Sampaolo, C M Vincitorio, Department of Neurosciences, School of Medicine, Federico II State University, Naples, Italy F Carrara, F Invernizzi, M Zeviani, Unit of Molecular Neurogenetics, Pierfranco and Luisa Mariani Center for the Study of Children's Mitochondrial Disorders, National Neurological Institute C. Besta, Milan, Italy \\ M De Caro, Division of Anesthesiology, Public General Hospital, Mercato San Severino (SA), Italy}

Correspondence to: $\operatorname{Dr} M$ Zeviani, Division of Molecular Neurogenetics, National Neurological Institute Carlo Besta, via Temolo 4, 20126 Milan, Italy; zeviani@tin.it

\section{REFERENCES}

1 Schwaz M. Vissing J. Paternal inheritance of mitochondrial DNA. New Engl J Med 2002;347:576-80.

2 Shanske S, Tang Y, Hirano M, Nishigaki Y, Tanji K, Bonilla E, Sue C, Krishna S, Carlo JR, Willner J, Schon EA, DiMauro S. Identical mitochondrial DNA deletion in a woman with ocular myopathy and in her son with pearson syndrome. Am J Hum Genet 2002;71:679-83.

3 Dubowitz V. Muscle biopsy: a practical approach. London: Bailliere-Tindall, 1985

4 Darley-Usmar VM, Rickwood D, Wilson MT, eds. Mitochondria, a practical approach. Washington DC: IRL Press, 1987

5 Andrews RM, Kubacka I, Chinnery PF, Lightowlers RN, Turnbull DM, Howell N. Reanalysis and revision of the Cambridge reference sequence for human mitochondrial. DNA Nat Genet 1999:2:147.

6 Anderson S, Bankier AT, Barrell BG, de Bruijn MHL, Coulson AR, Drouin J, Eperon IC, Nierlich DP, Roe BA, Sanger F, Schreier PH, Smith AJH, Staden R, Young IG. Sequence and organization of the human mitochondrial genome. Nature 1981:290:457-65.

7 Zeviani M, Moraes CT, DiMauro S, Nakase H, Bonilla E, Schon EA, Rowland LP. Deletions of mitochondrial DNA in Kearns-Sayre syndrome. Neurology 1988;38:1339-46.

8 Moraes CT, DiMauro S, Zeviani M, Lombes A, Shanske S, Miranda AF, Nakase H, Bonilla E, Werneck LC, Servidei S, Sensi A, Bonfatti A. 
Mitochondrial DNA deletions in progressive external ophthalmoplegia and Kearns-Sayre Syndrome. New Engl J Med 1989;320:1293-9.

9 Manfredi G, Vu T, Bonilla E, Schon EA, DiMauro S, Arnaudo E, Zhang L, Rowland LP, Hirano M. Association of myopathy with large-scale mitochondrial DNA duplications and deletions: which is pathogenic? Ann Neurol 1997;2:180-8.

10 Poulton J, Deadman ME, Bindoff L, Morten K, Land J, Brown G. Families of mtDNA re-arrangements can be detected in patients with $\mathrm{mtDNA}$ deletions: duplications may be a transient intermediate form. Hum Mol Genet 1993:2:23-30.

11 Poulton J, Morten KJ, Weber K, Brown GK, Bindoff L. Are duplications of mitochondrial DNA characteristic of Kearns-Sayre syndrome? Hum Mol Genet 1994;3:947-51.

12 Poulton J, Morten KJ, Weber K, Brown GK, Rotig A, Bindoff L. Duplications of mitochondrial DNA in Kearns-Sayre syndrome. Muscle Nerve 1995;3:S154-8.

13 Poulton J, Holt I. Mitochondrial DNA: does more lead to less? Nat Genet 1994:8:313-15.

14 Ballinger SW, Shoffner JM, Hedaya EV, Trounce I, Polak MA, Koontz DA, Wallace DC. Maternally transmitted diabetes and deafness associated with a $10.4 \mathrm{~kb}$ mitochondrial DNA deletion. Nat Genet 1992;1:11-15.
15 Martin Negrier ML, Coquet M, Moretto BT, Lacut JY, Dupon M, Bloch B, Lestienne P, Vital C. Partial triplication of mtDNA in maternally transmitted diabetes mellitus and deafness. Am J Hum Genet 1998;63:1227-32.

16 Dunbar DR, Moonie PA, Swingler RJ, Davidson D, Roberts R, Holt IJ. Maternally transmitted partial direct tandem duplication of mitochondrial DNA associated with diabetes mellitus. Hum Mol Genet 1993;2:1619-24.

17 Rötig A, Bessis J-L, Romero N, Cormier V, Saudubray J-M, Narcy P, Lenoir G, Rustin P, Munnich A. Maternally inherited duplication of the mitochondrial genome in a syndrome of proximal tubulopathy, diabetes mellitus and cerebellar ataxia. Am J Hum Genet 1992;50:364-70.

18 Bernes SM, Bacino C, Prezant TR, Pearson MA, Wood TS, Fournier P, FischelGhodsian N. Identical mitochondrial DNA deletion in mother with progressive external ophthalmoplegia and son with Pearson marrow-pancreas syndrome. J Pediatr 1993;123:598-602.

19 He L, Chinnery PF, Durham SE, Blakely EL, Wardell TM, Borthwick GM, Taylor RW, Turnbull DM. Detection and quantification of mitochondrial DNA deletions in individual cells by real-time PCR. Nucleic Acids Res 2002:30 e68.

20 Poulton J, Turnbull DM 74th ENMC international workshop: mitochondrial diseases 19-20 november 1999, Naarden, the Netherlands. Neuromuscul Disord 2000;10:460-2.

\section{$\mathrm{ECHO}$}

Brain cysts associated with mutation in the Aristaless related homeobox gene, ARX

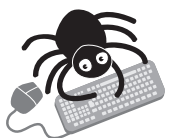

Please visit the Journal of

Medical

Genetics website [www. jmedgenet. com] for a link to the full text of this article. 24bp duplication mutation in exon 2 of the $A R X$ gene has been identified in a 72 year
old man with X-linked mental retardation, a spastic ataxic gait, and dystonia of hand
and facial muscles. MRI examination showed cerebrospinal fluid filled cystic cavities
surrounded by gliosis in both cerebral and cerebellar hemispheres.

As the cysts were lying at the outer margins of the brain substance, infarcts were considered as a cause but the largest lesion was too extensive to represent small vessel occlusion. In addition, the lesions were outside the watershed area and there was no surrounding parenchymal retraction.

The patient had no history of acute neurological impairment and no identifiable risk factors for cerebrovascular disease. Other members of his family had various neurological impairments including infantile spasms, epilepsy, spasticity, and cerebellar ataxia. One relative had bilateral cerebral and cerebellar cysts.

The investigators conclude the most likely explanation for the cystic cavities are abnormalities of the developing fetal brain caused by the $A R X$ mutation. The gene is widely expressed in the brain and has been shown to play an important role in neuronal proliferation and interneuronal migration and proliferation in a knockout mouse model.

A Journal of Neurology, Neurosurgery, and Psychiatry 2003;74:536-538. 